$\xi=1$ 圆

\title{
Neural learning: price prediction for non-agricultural commodities using back propagation network
}

\author{
Hudson Arul Vethamanikam G*', Mary Kiruba Rani $V^{2}$, Joel Jebadurai $D^{3}$ \\ ${ }^{1}$ Assistant Professor, School of Management, CMR University \\ ${ }^{2}$ MANF-Doctoral research Scholar,Department of Computer Science, Alagappa University \\ ${ }^{3}$ Assistant Professor, School of Management ,Acharya Institute of Graduate Studies \\ *Corresponding author E-mail:hudsonsukisam@gmail.com
}

\begin{abstract}
Neural Network is relatively superlative in predicting economic data. The concept for econometric research contracts with predicting the price variation of non-agricultural commodities. With a focus on gold, silver, aluminium, lead, zinc, natural gas, crude oil the systematic learning for finding the price growth is the aim of this research. The methodology implemented deals with neural network back propagation for training and testing. The input data are learned with 0.2 learning rate and trained until minimization of error. The price of describing commodities from 2007 to 2016 year and it is predicted that within this period the importing commodities get profit up to $77 \%$ with combination of gold, silver, aluminium and crude oil are sold as equally. Systematically, the goods are learned easily in the proposed methodology and the error rate is minimized at the lowest.
\end{abstract}

Keywords:Neural Network; Back Propagation; Non-Agricultural Commodities; Price; Profit Estimation.

\section{Introduction}

Artificial Neural Network (ANN) is applied to other areas relatively for solving non-agricultural commodities. It is a nonlinear approach for interpolation abilities in the system learning attributes for prediction. Technical analysis to find the growth of nonagricultural commodities using historical data, observe the behavior and formulate rules for prediction. Extracting valuable information through automatic learning of the system is done through neural network.

It is an artificial intelligent technique for finding patterns related to input data and corresponding output targets in complex data types. After testing and training with data it can work with new data. This computational model is suited for modelling the nonagricultural commodities data which are nonlinear in structure and complexity.

Since neural network is a robust system and fault tolerant it can fulfil the pattern even though the data are incomplete are noisy in nature. Thus, the raw data on commodities are processed and the time series of growth of each commodity is generalized.

The non-agricultural commodities are Gold, Silver, Aluminium, Copper, Lead, Nickel, Zinc, Crude Oil and Natural Gas.

Grading the non-agricultural commodities using machine vision along with neural network gives more advantages over conventional devices. Neural networking algorithm extracts higher level information from the input signals and improves in classification and prediction. When training the input data, different grading is recognized for different products.

This work is general information on analysis of historical growth for non-agricultural commodities. The goal is to make the system learn the attributes and make predictions on the growth of various products. The benefits of using neural network algorithm are to maximize the accuracy for quite desirable and usable predictable tool.

Table 1:Non- Agricultural Data of Commodities for 10 Years

\begin{tabular}{llllllll}
\hline Year & Gold & Silver & Aluminium & Copper & Lead & Zinc & Crude Oil \\
\hline 2007 & 15.55 & 15.54 & 11.02 & 14.95 & 5.26 & 14.43 & 14.74 \\
2008 & 16.63 & 15.77 & 10.75 & 15.09 & 13.42 & 14.22 & 15.36 \\
2009 & 16.86 & 15.73 & 10.73 & 15.06 & 13.04 & 13.54 & 15.89 \\
2010 & 16.69 & 16.17 & 13.48 & 15.84 & 14.73 & 15.02 & 15.83 \\
2011 & 16.81 & 17.24 & 13.66 & 16.20 & 15.07 & 15.17 & 16.51 \\
2012 & 17.31 & 17.44 & 13.98 & 16.49 & 15.11 & 14.88 & 16.86 \\
2013 & 17.32 & 17.36 & 14.80 & 16.15 & 15.06 & 15.33 & 16.90 \\
2014 & 16.36 & 16.21 & 13.60 & 14.97 & 14.09 & 14.25 & 15.98 \\
2015 & 16.33 & 15.97 & 14.12 & 15.23 & 14.48 & 14.71 & 16.30 \\
2016 & 16.17 & 15.60 & 13.95 & 14.96 & 14.06 & 14.89 & 16.89 \\
\end{tabular}

Further sections in this work consist of section 2 deals with existing work related to the theme; section 3 explains about the algo-

rithm and systematic learning of input data; section 4 constructs research in the system and analysis, performed through neural 
network; section 5 concludes with the performance in growth of non-agricultural commodities.

\section{Existing methodology}

This section provides an overview of existing work on sharing the knowledge in the stated work. It includes the findings and previous studies from different authors.

S. Kumar Chandar, M. Sumathi, et al [1], proposed a methodology for predicting the Gold rate for a future based on Extreme Learning Machine(ELM) in back propagation networks. Soft computing techniques prove to be the best method for predicting profit in rate.

Ching-Hwang Wang, et al [2], developed an iterative model for learning performance in calculating the data into training and testing period. The decision-making process proposes the Individua Inference Adjusting Learning Rate technique (IIALR).

Massimo Panella, Francesco Barcellona, et al [3], considered a prediction model provides sequences to predict the market data. A linear model Least Square Error (LSE) approximation, Radial Basis function, Back propagation Neural Network are implemented to find the price dynamics of commodities.

Pushpa Mohan, et al, [6] proposed a methodology for Crop Cost Forecasting using Artificial Neural Network with feed forward back propagation method for Mysore Region with Artificial Neu- ral Networks and feed forward back propagation networks. The paper enhances Mean Square Error and provide a rate for crops. Andres M. Ticlavilca, et al [7] developed Forecasting Agricultural Commodity Prices Using Multivariate Bayesian Machine Learning Regression for the purpose of analyzing commodity price.

\section{Proposed methodology}

Neural Network is a class of input data and output target capable of memorizing and systematic learning for automatic prediction. In addition, with mathematical analysis non-agricultural commodities are learned by the system for fast prediction.

\subsection{Data}

The data set for proposed methodology consists of nonagricultural commodities over ten years. The chosen commodities are gold, silver, aluminium, copper, lead, nickel, zinc, crude oil and natural gas. The dataset consists of 70 percent training set and 30 percent testing set values. Table 2 data show the price variation for ever year. This knowledge is implemented in the hidden layer of neural network structure.

Table 2: Variation of Price for Commodities

\begin{tabular}{|c|c|c|c|c|c|c|c|}
\hline Gold & Silver & Aluminium & Copper & Lead & Zinc & Crude Oil & Natural Gas \\
\hline 1.08 & 0.23 & -0.27 & 0.14 & 0.16 & -0.21 & 0.62 & -1.29 \\
\hline 0.23 & -0.04 & -0.02 & -0.03 & -0.38 & -0.68 & 0.53 & 0.91 \\
\hline-0.17 & 0.44 & 1.75 & 0.78 & 1.69 & 1.48 & -0.06 & 2.35 \\
\hline 0.5 & 0.2 & 0.38 & 0.29 & 0.04 & -0.29 & 0.35 & 0.21 \\
\hline 0.01 & -0.08 & 0.82 & -0.34 & -0.05 & 0.45 & 0.04 & 0.74 \\
\hline-0.96 & -1.15 & -1.2 & -1.18 & -0.97 & -1.08 & -0.92 & 0.06 \\
\hline-0.03 & 0.24 & 0.52 & 0.26 & 0.39 & 0.46 & 0.32 & -0.83 \\
\hline-0.16 & 0.37 & -0.17 & -0.27 & -0.42 & 0.18 & 0.19 & -0.41 \\
\hline
\end{tabular}

\subsection{Self-learning neural network}

In self training neural network system, inaccurate information to the next phase form more error in classification. To reduce the error, the base classifiers need to learn the accurate data from the training data set. Thus, the proposed methodology is processed for attaining accurate results in learning. The largest number of label data is generalized to make the system learn. Initially randomly generated weights are used for learning process. The pre-training makes the learning better in prediction.

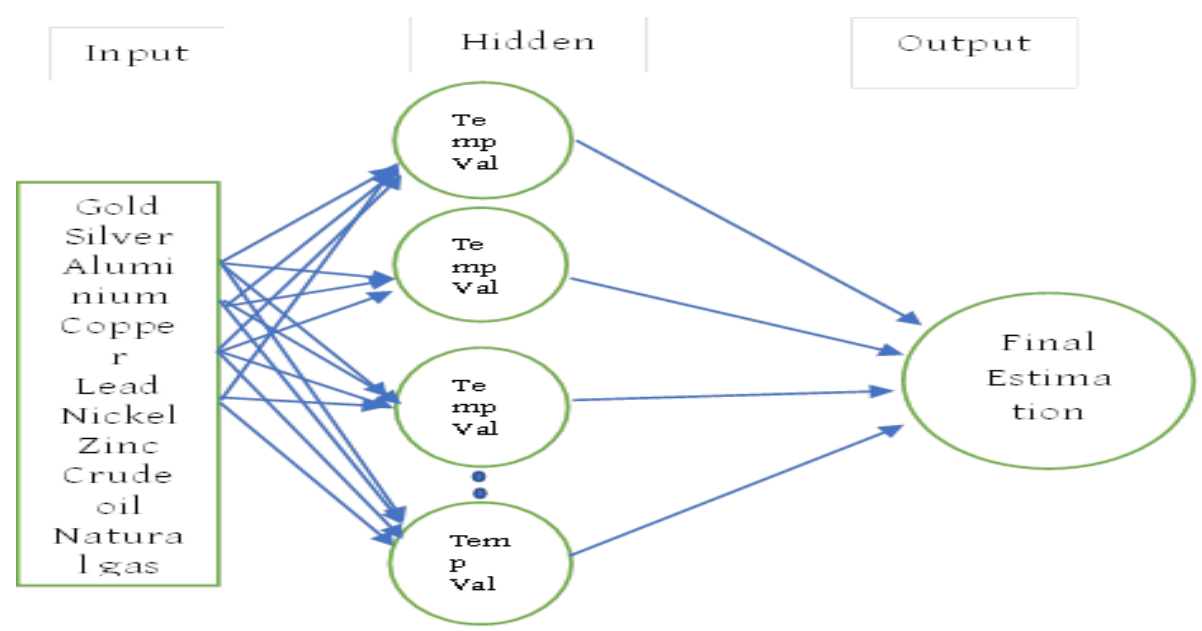

Fig. 1: Structure of Neural Network Learning. 


\subsection{Back propagation network}

To reduce the error rate of learning attributes back propagation neural network evolves. The steps that involve in propagating the network into backward class is as follows

Algorithm:

Step 1: Weight Initialization

Initially assign random values to each input vector.

Step 2:These random values are multiplied with the input vector $\mathrm{X}_{\mathrm{i}}$ and pass the signal out.

(i.e.) Hidden unit

Step 3: Hidden unit is summed with the input signal

$\mathrm{Z}_{\mathrm{i}}=\mathrm{V}_{\mathrm{i}}+\sum \mathrm{X}_{\mathrm{i}} \mathrm{W}_{\mathrm{i}}$

Where, $V_{i}$ is hidden unit value, $W_{i}$ is the weight vector, $X_{i}$ is the input vector. And to process it calls the Activation function.

$\mathrm{Z}_{\mathrm{j}}=\mathrm{f}\left(\mathrm{Z}_{\mathrm{i}}\right)$

Step 4: Each output unit sums the weighted input signals $Y_{k}$,

$\mathrm{k}=1 \ldots \mathrm{N}$

$\mathrm{Y}_{\mathrm{k}}=\mathrm{W}_{\mathrm{ij}}+\sum \mathrm{Z}_{\mathrm{i}}+\mathrm{W}_{\mathrm{i}}$

And calculate output signals based on this activation function.

$\mathrm{Y}_{\mathrm{fk}}=\mathrm{f}\left(\mathrm{Y}_{\mathrm{k}}\right)$

Step 5: Each output vector receives a pattern corresponding to its input unit. And if it is not met by the target vector error function is calculated as

$\delta_{\mathrm{k}}=\left(\mathrm{t}_{\mathrm{k}}-\mathrm{Y}_{\mathrm{k}}\right) \mathrm{f}\left(\mathrm{Y}_{\mathrm{fk}}\right)$

Calculates its bias correction term $\Delta \mathrm{W}_{\mathrm{ok}}=\alpha \delta_{\mathrm{k}}$ and sends $\delta_{\mathrm{k}}$ two units to the next layer.

Step 6: This error function again updated in weight and bias hidden unit.

Step 7: Stop the condition till minimization of the errors.

Training:

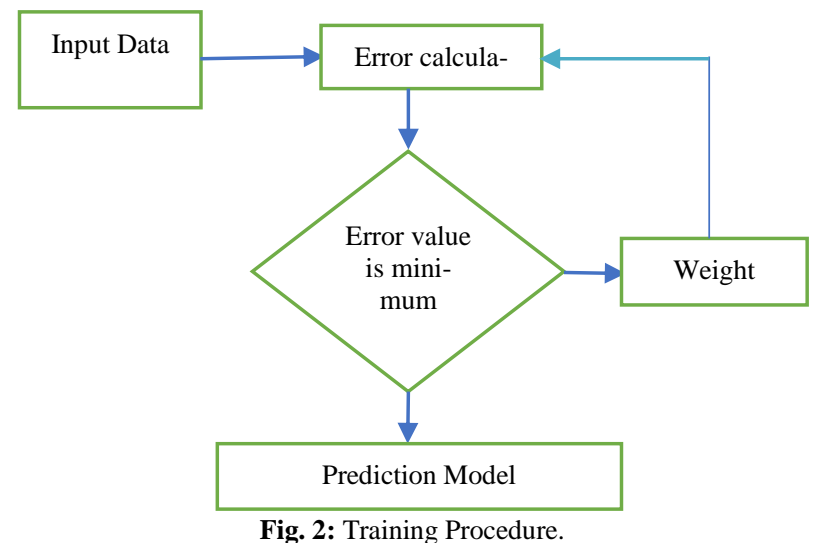

In the training process, the input data is multiplied with the randomly generalized weight, which ranges from 0 to 1 . The multiplied output performs the error calculation. If the value is minimized, then the process stops as it iterates until convergence.
Testing:

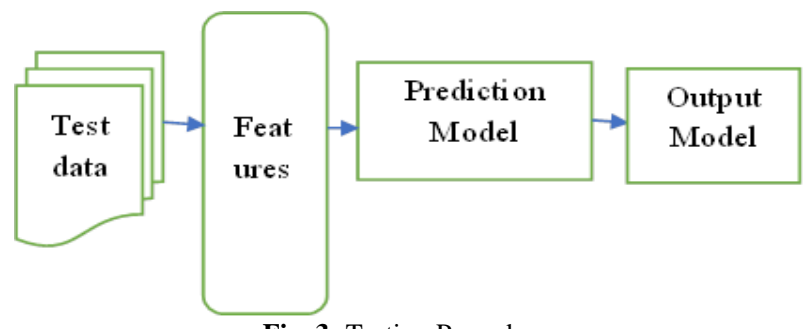

Fig. 3: Testing Procedure.

In the testing phase, $30 \%$ of raw data are passed into the network by separating the features. These data were processed with already trained data and check out for price prediction.

Back propagation initially determines the topology of the network by initiating the weights and threshold. Comparing with optimal weight and threshold, calculate the errors. Modify the weight till the error is minimized. The network used for this research is threelayer network. Thus, the error values propagate backward until convergence.

\subsection{Evaluation metrices}

\subsubsection{Mean}

The Mean value is used to find the yearly average of each commodity. Increase in price prediction depends upon the average price growth in every year. The mean value calculation is as follows:

\section{$\mathrm{X}=1 /(\mathrm{Y}) \sum \mathrm{xy}$}

Where, $\mathrm{X}$ is the MEAN value,

$\mathrm{Y}$ is the number years for prediction.

$\mathrm{x}$ is the difference values of every month.

$y$ is the difference values of every year.

The mean values give the average increase percent of nonagricultural commodities.

\subsubsection{Median}

The Median value is used to find the highest possible value in growth difference. Logically, median value among the ordered list is the bridge for finding gain and loss in the price calculation. This value will be useful for importing or exporting.

$\mathrm{MDN}=(\mathrm{M} / \mathrm{N})$ th term is the median

\subsubsection{Mode}

This kind of quality measurement is used to find the values which are occurring frequently. The process is used to find the maximum rate of any product.

\subsubsection{Standard deviation (SD)}

Standard Deviation is the principle of measuring closing prices for instance. Values of standard deviation will be higher if the difference between the closing price and the average price is larger. The formula for Standard Deviation calculation is by the following steps

1) Calculate the mean of all prices.

2) Find the deviation of each period by subtracting the average and closing price.

3) Find the square of each deviation.

4) Sum the deviation and divide it with total number of years.

5) The standard deviation is $\mathrm{SD}=\sqrt{ }\left(\sum(\mathrm{X}-\mathrm{X} 1) 2 /(\mathrm{N}-1)\right)$. 


\subsubsection{Mean square error (MSE)}

Mean Square Error (MSE) tells how close the regression lines from input to output. If the distance is less, then the desired values are suitable for prediction, otherwise the data should be propagated backward until error comes minimum. The formula for MSE is

$\mathrm{MSE}=\frac{1}{n} \sum_{i=1}^{n}(Y-Y 1) 2$

\section{Analysis and findings}

The optimization of price prediction over several commodities is challenged with a back propagation neural network. This algorithm makes the electronic system learn the data and reports the supervised output. The following tables show the analysis report.

\begin{tabular}{lllll}
\hline S1. No & Commodities & Mean & MSE & Probability \\
\hline 1 & Agricultural crop & 1256 & 1.21 & $>1$ \\
2 & Agricultural crop & 1288 & 0.85 & $<1$ \\
3 & Non- Agricultural & 14.82 & 0.44 & $<0.5$ \\
\hline
\end{tabular}

Table 3:The Error Generated for Each Commodity and Mean Value of Price

\begin{tabular}{llll}
\hline S1. No & Commodity & MSE & MEAN \\
\hline 1 & Gold & 0.12 & 12.46 \\
2 & Silver & 0.54 & 16.30 \\
3 & Aluminium & 0.24 & 13.00 \\
4 & Copper & 0.01 & 15.49 \\
5 & Lead & 0.95 & 13.43 \\
6 & Zinc & 0.46 & 14.54 \\
7 & Crude Oil & 0.51 & 16.08 \\
8 & Natural Gas & 0.73 & 14.33 \\
\hline
\end{tabular}

Table 4:Price Growth Chart Over 10 Years of Non-Agricultural Commodities

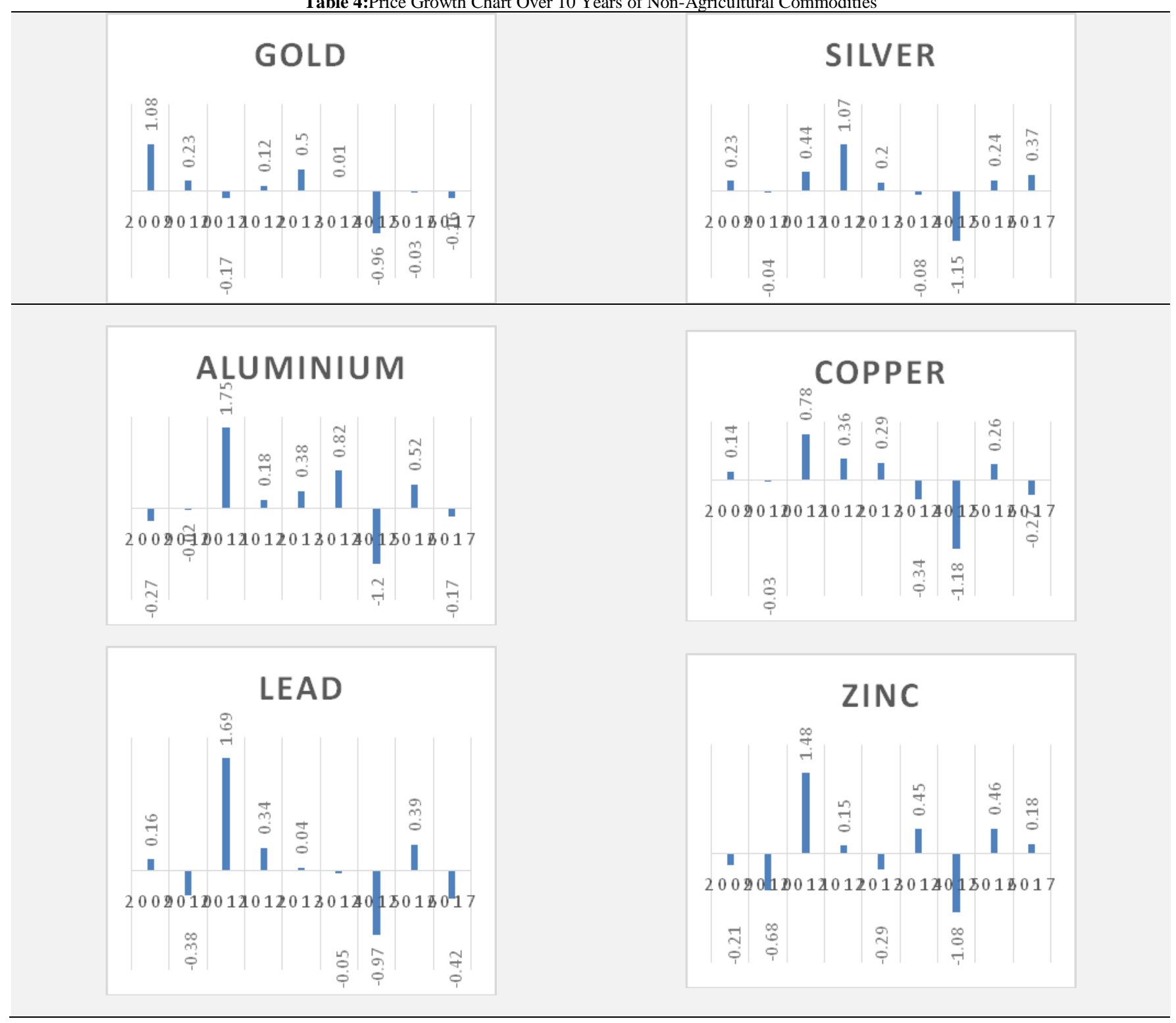




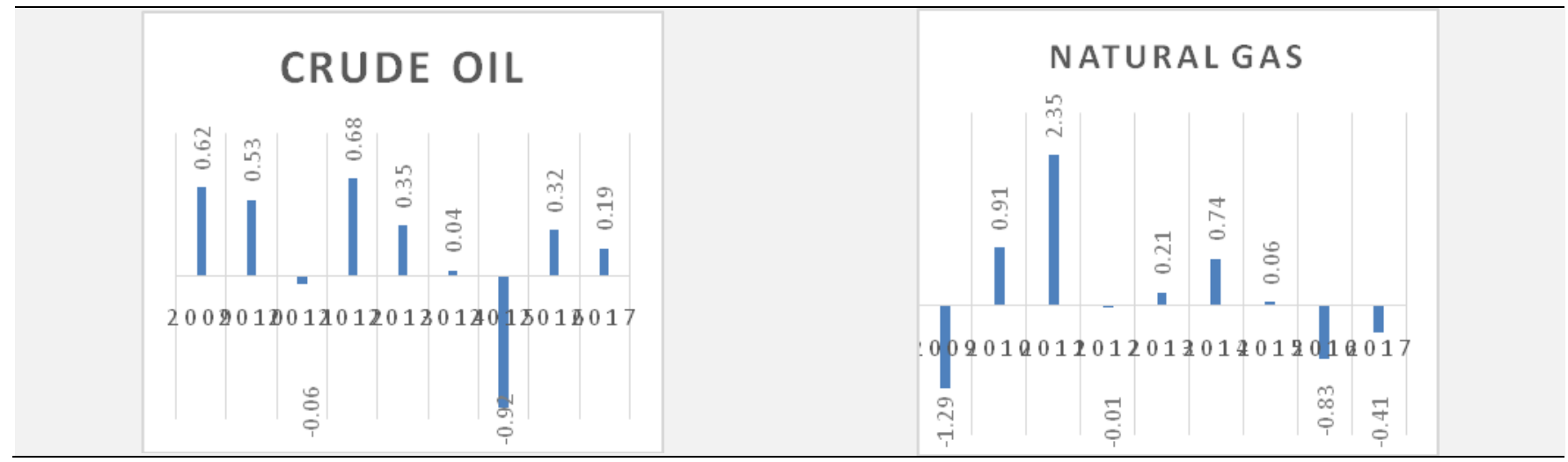

From table 4 the commodities from the period 2009 to 2017, it is analyzed the price growth is high for gold, silver, copper and crude oil compared to zinc, Aluminium, Natural gas, Lead. The growing ability of such highly growing commodities integrates with $77 \%$ with 9 years of span. This gives clear opportunity for importing gold, silver, aluminium and crude oil as a combination for yielding best profits.

The probability for best price estimation findings is less than 0.5 for non-agricultural commodities. Thus, the algorithm for nonagricultural finding is best compared to other methods through neural network approach.

The neural network is implemented for self-training the system to find the growth percentage of every commodities. Learning leads the system to automatically find the variation of price between the years. For example, the price growth of gold from 2009 to 2013 is good in growth and loss for next 2 years and again it grew high. Thus, compared with 10 years of data, prediction through the back propagation neural network the proposed methodology has high efficiency in importing commodities for economic growth in our country.

\section{Conclusion}

Non-agricultural commodity prices are both high and low in discrepancy for the past few decades. However, the increase in price as well as the growth of each product is unpredictable. A systematic learning for the growth in price is a challenging task. Neural network plays a vital role in systematic learning for many data. It is possible that the commodities, gold, silver, aluminium, copper, lead, zinc, crude oil, natural gas is trained and tested with a back propagation neural network.

The learning rate with 0.2 possibly good to predict the theme ideas. In terms of Mean Square Error (MSE), it is less, and it yields better results compared to other. The Neural network has learned and fault tolerant capabilities. Thus, finding the best solution for the global is acceptable. The research work deals with finalizing that using gold, silver, copper and crude oil compared to zinc, Aluminium, Natural gas, Lead. This result would be useful for business people who earn a lot. The growth of prize would increase $77 \%$ over every ten years of span.

\section{References}

[1] S. Kumar Chandar, M. Sumathi, S.N. Sivanadam," Forecasting Gold Prices Based On Extreme Learning Machine", International Journal Of Computers Communications \& Control ISSN 1841 9836, 11(3):372-380, June 2016.

[2] Ching-Hwang Wang, Chih-Han Kao, Wei-Hsien Lee," A new interactive model for improving the learning performance of back propagation neural network", Automation in Construction 16 (2007) 745-758.https://doi.org/10.1016/j.autcon.2006.12.007.

[3] Massimo Panella, Francesco Barcellona Valentina Santucci and Rita L. D'Ecclesia ," Neural Networks to Model Energy Commodity Price Dynamics".

[4] V. Mary Kiruba Rani, S.S. Dhenakran," A Mathematical Modelling for Quality Based Ultrasound Breast Cancer Image Using Colour-
Properties", Australian Journal of Basic and Applied Sciences, 10(2) Special 2016, Pages: 44-51.

[5] Girish K. Jha and Kanchan Sinha," Agricultural Price Forecasting Using Neural Network Model: An Innovative Information Delivery System", Agricultural Economics Research Review Vol. 26 (No.2) July-December 2013 pp 229-239.https://doi.org/10.1007/s40003013-0068-4.

[6] G. Hudson Arul Vethamanikam, S. Rajamohan," Unit Root Test Of Selected Non-Agricultural Commodities And Macro Economic Factors In Multi Commodity Exchange Of India Limited", International Journal of Advanced Research in Management and Social Sciences, ISSN: 2278-6236,Impact Factor: 6.943.

[7] Pushpa Mohan, Kiran KumariPatil, "Crop Cost Forecasting using Artificial Neural Network with feed forward back propagation method for Mysore Region", International Journal of Innovative Research in Science, Engineering and Technology, Vol. 6, Issue 4 April 2017. 